\title{
Contração volumétrica e cinética de secagem de fatias de banana variedade Terra ${ }^{1}$
}

\author{
Anna Letícia Moron Pereira Leite ${ }^{2}$, Fabrício Schwanz da Silva ${ }^{3}$, \\ Alexandre Gonçalves Porto ${ }^{3}$, Diego Piasson ${ }^{3}$, Philipe dos Santos ${ }^{2}$
}

\begin{abstract}
Volumetric contraction and drying kinetics of Musa sapientum slices

Drying is a method employed in food preservation, which influences the preservation time, quality and durability of these products. This study aimed to characterize the drying kinetics and evaluate the shrinkage of Musa sapientum slices, under temperatures of $40-60^{\circ} \mathrm{C}$. The mathematical models used to analyze drying were diffusion approximation, two terms, Midilli \& Kucuk and Thompson, while the ones used to evaluate shrinkage were Lozano, McMinn \& Magee, Rovedo, Suarez \& Viollaz and Ratti. The model that best fitted the experimental drying data was Midilli \& Kucuk, for presenting the best coefficient of determination $\left(\mathrm{R}^{2}\right)$ and the lowest average relative error $(\mathrm{P})$ and estimate standard deviation (SE). For shrinkage, the best model was Ratti, due to its higher $\mathrm{R}^{2}$ and lower $\mathrm{P}$ and $\mathrm{SE}$. The volume reduction (VR) presented an inverse relation with temperature. For temperatures of $40{ }^{\circ} \mathrm{C}, 50{ }^{\circ} \mathrm{C}$ and $60{ }^{\circ} \mathrm{C}$, the VR estimates with pachymeter were respectively $21.65 \%$, $19.46 \%$ and $17.74 \%$, while the estimates with image were $27.74 \%, 24.08 \%$ and $22.50 \%$.
\end{abstract}

KEY-WORDS: Musa sapientum; dehydration; mathematical models.

\section{INTRODUÇÃO}

O Brasil destaca-se, dentre os países em desenvolvimento, como um dos maiores produtores mundiais de frutas, sendo a perda de produtos in natura estimada em torno de $50 \%$ (Paraná 2012), podendo estar relacionada à falta de tecnologias adequadas de pós-colheita, transporte e armazenamento.

Para evitar perdas na pós-colheita, as frutas necessitam de temperaturas especiais para a sua conservação, como ocorre, por exemplo, no processo de refrigeração (Chitarra \& Chitarra 1990, Brunini et al. 2002).

\section{RESUMO}

A secagem é um método empregado na conservação de alimentos, influenciando no tempo de preservação, qualidade e durabilidade desses produtos. Este trabalho objetivou caracterizar a cinética de secagem e avaliar o encolhimento de fatias de banana variedade Terra, sob temperaturas de secagem de $40-60{ }^{\circ} \mathrm{C}$. Os modelos matemáticos utilizados para a análise da secagem foram aproximação da difusão, dois termos, Midilli \& Kucuk e Thompson e, para o encolhimento, Lozano, McMinn \& Magee, Rovedo, Suarez \& Viollaz e Ratti. O modelo que melhor se ajustou aos dados experimentais de secagem foi o de Midilli \& Kucuk, por apresentar o melhor coeficiente de determinação $\left(\mathrm{R}^{2}\right)$ e o menor erro médio relativo $(\mathrm{P})$ e desvio padrão da estimativa (SE). Já para o encolhimento, o melhor modelo foi o de Ratti, por apresentar maior $\mathrm{R}^{2}$ e menores $\mathrm{P}$ e SE. A redução de volume (RV) apresentou comportamento inversamente proporcional, em relação à temperatura. Para as temperaturas de $40^{\circ} \mathrm{C}, 50^{\circ} \mathrm{C}$ e $60{ }^{\circ} \mathrm{C}$, a RV foi, respectivamente, de $21,65 \%, 19,46 \%$ e $17,74 \%$, quando avaliada com paquímetro, e de $27,74 \%, 24,08 \%$ e $22,50 \%$, quando avaliada com imagem.

PALAVRAS-CHAVE: Musa sapientum; desidratação; modelos matemáticos.

Técnicas para a preservação e diminuição de prejuízos causados a produtos in natura são estudadas há anos. O desafio é preservar os alimentos, mantendo valores nutricionais semelhantes aos originais, pois os consumidores estão cada vez mais exigentes. Em níveis industriais, tem-se buscado o desenvolvimento de operações que minimizem os custos e os efeitos adversos do processamento. Logo, torna-se de grande interesse o estudo de operações de processamento para produtos à base de frutas tropicais (Pontes et al. 2007).

No Brasil, o consumo e a produção de frutas tropicais ocupam o terceiro lugar mundial. A banana

1. Trabalho recebido em jun./2014 e aceito para publicação em abr./2015 (http://dx.doi.org/10.1590/1983-40632015v4530270).

2. Universidade Estadual de Campinas (Unicamp), Faculdade de Engenharia de Alimentos, Departamento de Engenharia de Alimentos, Campinas, SP, Brasil.E-mails: anna_mpl@hotmail.com, philipe.dsn@gmail.com.

3. Universidade do Estado de Mato Grosso (Unemat), Departamento de Engenharia de Alimentos, Barra do Bugres, MT, Brasil. E-mails: fabricio@unemat.br, agporto@unemat.br,diegopiasson@hotmail.com. 
é uma fruta de destaque, representando $15,9 \%$ do volume total da fruticultura produzida no País (Paraná 2012), estando presente na dieta dos brasileiros por fornecer quantidades significativas de micronutrientes, como vitaminas e minerais. Porém, o Brasil também é o País com maior índice de desperdício dessa fruta (Sousa 2002, Jesus et al. 2004).

Devido ao elevado índice de desperdício e perecibilidade, essa fruta carece de técnicas que aperfeiçoem o seu armazenamento. A desidratação tem como objetivo eliminar um líquido volátil (água) contido na matéria-prima, por meio da técnica de evaporação, proporcionando a conservação desse alimento por mais tempo (Dantas et al. 2009).

Durante a desidratação, o produto pode sofrer redução em seu volume (encolhimento), devido a alterações na microestrutura do material fresco, como o aumento de cavidades, dentre outras transformações causadas pelo estresse térmico, e, principalmente, pela remoção de umidade. As alterações estruturais dependem, também, do formato do produto estudado (Katekawa 2006, Borges et al. 2008).

A retirada parcial ou total da água livre impede a ocorrência de reações bioquímicas responsáveis pela deterioração, implicando em maior preservação do fruto e impedindo, assim, a ocorrência de reações bioquímicas responsáveis pela deterioração. Essa remoção de água se faz necessária para evitar os danos causados por micro-organismos, aumentando, então, a vida de prateleira do produto (Drouzas \& Shubert 1996, Park et al. 2001).

O estudo da secagem de produtos sólidos em camada delgada é utilizado para a determinação experimental dos parâmetros que caracterizam a operação, obtendo-se, assim, um melhor domínio do processo, como, também, maior conhecimento dos fundamentos e mecanismos envolvidos (Batista 2004).

O conhecimento da umidade de equilíbrio é de suma importância para os processos de transformação, preservação e armazenamento de alimentos. A estimativa da umidade de equilíbrio é necessária para a avaliação da cinética de secagem, utilização dos modelos matemáticos e determinação da constante de secagem $(\mathrm{k})$, difusividade efetiva de massa $\left(D_{\text {ef }}\right)$ e energia de ativação $\left(E_{\mathrm{a}}\right)$ (Batista 2004, Silva et al. 2009).

A velocidade de troca de calor entre o produto e o ar pode ser analisada por meio da $\mathrm{D}_{\text {ef }}$, nas diferentes temperaturas do processo (Park et al. 2001).
O coeficiente de difusão mássica $\left(\mathrm{D}_{\mathrm{ef}}\right)$ é considerado importante propriedade de transporte, a qual compreende os efeitos de todos os fenômenos que possam vir a interferir na migração da água (Reis et al. 2011).

A energia de ativação ( $E_{\mathrm{a}}$ ) é definida como a energia necessária para romper as barreiras que as moléculas de água encontram ao migrarem do interior para a superfície do alimento, durante o processo de desidratação (Sharma \& Prasad 2004).

O presente estudo objetivou caracterizar a cinética de secagem e avaliar o encolhimento de fatias de banana da variedade Terra (Musa sapientum, Linneo).

\section{MATERIAL E MÉTODOS}

Os experimentos foram realizados no Laboratório de Engenharia e Processamento Agroindustrial da Universidade do Estado de Mato Grosso (Unemat), em Barra do Bugres (MT), de agosto de 2010 a outubro de 2011.

A matéria-prima foi adquirida de produtores da microrregião de Tangará da Serra (MT). As frutas, ao chegarem ao laboratório, foram higienizadas, descascadas e fatiadas (fatiador Kenmore, modelo $\mathrm{n}^{\mathrm{o}} \mathrm{Z} 3036$, com espessura de $1 \mathrm{~mm}$ ).

Os ensaios de secagem foram realizados em duplicatas, utilizando-se secador descontínuo de bandejas, em escala de bancada, com temperaturas do ar de secagem de $40{ }^{\circ} \mathrm{C}, 50{ }^{\circ} \mathrm{C}$ e $60^{\circ} \mathrm{C}$ e velocidade de $1 \mathrm{~m} \mathrm{~s}^{-1}$, garantindo que a resistência à transferência de massa fosse controlada pela migração de água, no interior do material. As fatias foram colocadas em bandejas inoxidáveis de fundo maciço, as quais foram devidamente pintadas de preto, para facilitar a captura de imagens.

Para a realização das pesagens, foi utilizada balança eletrônica semianalítica (OHAUS Modelo AR3130), com precisão de 0,01 g. A aquisição dos valores dos diâmetros foi realizada utilizando-se paquímetro digital (marca Starrett), com precisão de 0,001 mm. Já para a obtenção das imagens, foi utilizada uma câmara CCD profissional (marca NITRIX, lente para CFTV de 3,5-8,0 mm).

As amostras foram retiradas do secador, para verificações de pesos e medidas, sendo, inicialmente, realizadas em intervalos de 15 minutos, nas primeiras 3 horas, e, posteriormente, de 30 minutos, até completar o período de 12 horas de secagem. Após 12 horas, para as amostras desidratadas a $40{ }^{\circ} \mathrm{C}$, continuou-se a pesagem, até peso constante, enquanto, para as amos- 
tras desidratadas a $50{ }^{\circ} \mathrm{C}$ e $60{ }^{\circ} \mathrm{C}$, foram realizadas pesagens em intervalos de 2 horas, até peso constante.

Durante o processo de secagem, a razão de umidade é essencial para descrever os diferentes modelos matemáticos (Roca et al. 2008). Sendo assim, a razão de umidade (RU), durante a secagem, nas diferentes condições de ar, foi determinada por meio da expressão $\mathrm{RU}=\left(\mathrm{U}^{*}-\mathrm{Ue}^{*}\right) /\left(\mathrm{Ui}^{*}-\mathrm{Ue}^{*}\right)$, em que $\mathrm{RU}$ = razão de umidade do produto, adimensional; $\mathrm{U}^{*}=$ teor de água do produto (b.s.); $\mathrm{U}_{\mathrm{e}}^{*}=$ teor de água de equilíbrio do produto (b.s.); $\mathrm{U}_{\mathrm{i}}^{*}=$ teor de água inicial do produto (b.s.).

São muitos os modelos matemáticos empíricos utilizados por pesquisadores para predizer o fenômeno da secagem de produtos agrícolas (Afonso Júnior \& Corrêa 1999, Akpinar et el. 2003, Ertekin \& Yaldiz 2004, Lahsasni et al. 2004). A Tabela 1 apresenta os modelos utilizados neste estudo.

O encolhimento ( $\mathrm{S}$ ) é frequentemente representado pela equação $\mathrm{S}=\mathrm{V} / \mathrm{V}_{0}$, que relaciona o volume de uma amostra a um determinado tempo de secagem e o volume da amostra no tempo inicial, representando uma alteração dimensional do volume, área e espessura, sendo $\mathrm{S}=$ encolhimento (adimensional); $\mathrm{V}=$ volume da amostra em um determinado tempo $\left(\mathrm{m}^{3}\right) ; \mathrm{V}_{0}=$ volume inicial da $\operatorname{amostra}\left(\mathrm{m}^{3}\right)$.
Os volumes iniciais e de equilíbrio foram obtidos multiplicando-se a área planimetrada pela espessura $\left(\mathrm{L}_{0}\right)$ da amostra, a qual se manteve a $1 \mathrm{~mm}$, durante todo o processo de secagem. Utilizando-se a média dos três diâmetros (Figura 1a) aferidos com paquímetro digital, foi determinada a área das amostras.

As imagens foram captadas por meio de uma estrutura experimental (Figura 1b). O software Matlab $^{\circledR}$ converteu as imagens, reconhecendo apenas as escalas de cores preto e branco, sendo a cor branca referente à fatia do fruto, gerando, em seguida, os valores da área das fatias, no decorrer do processo de secagem.

Para predizer o fenômeno do encolhimento, durante a secagem, utilizaram-se equações (Tabela 2) empíricas lineares e não lineares, ajustadas aos dados experimentais por meio de regressões matemáticas.

Para o ajuste dos modelos matemáticos de secagem e encolhimento, foram realizadas análises de regressão não linear, pelo método Gauss-Newton. O grau de ajuste de cada modelo considerou a magnitude do coeficiente de determinação $\left(\mathrm{R}^{2}\right)$, do desvio padrão da estimativa (SE) e do erro médio relativo (P). O erro médio relativo e o desvio padrão da estimativa, para cada um dos modelos de secagem e encolhimento, foram calculados conforme as expressões abaixo, respectivamente:

Tabela 1. Modelos matemáticos utilizados para estimar as curvas de secagem.

\begin{tabular}{lll}
\hline \multicolumn{1}{c}{ Designação do modelo } & \multicolumn{1}{c}{ Modelo } & \multicolumn{1}{c}{ Referência } \\
\hline Aproximação da difusão & $\mathrm{RU}=\mathrm{a} \exp (-\mathrm{kt})+(1-\mathrm{a}) \exp (-\mathrm{kbt})$ & Menges \& Ertekin $(2006)$ \\
Dois termos & $\mathrm{RU}=\mathrm{a} \exp \left(-\mathrm{k}_{0} \mathrm{t}\right)+\mathrm{b} \exp \left(-\mathrm{k}_{1} \mathrm{t}\right)$ & Dandamrongrak et al. (2002) \\
Midilli \& Kucuk & $\mathrm{RU}=\mathrm{a} \exp \left(-\mathrm{kt}^{\mathrm{n}}\right)+\mathrm{bt}$ & Midilli et al. $(2002)$ \\
Page & $\mathrm{RU}=\exp \left(-\mathrm{kt}^{\mathrm{n}}\right)$ & Midilli et al. $(2002)$ \\
Thompson & $\mathrm{RU}=\exp \left(\left(-\mathrm{a}-\left(\mathrm{a}^{2}+4 \mathrm{bt}\right)^{0,5}\right) / 2 \mathrm{~b}\right)$ & Erterkin \& Yaldiz $(2004)$ \\
\hline RU: razão de umidade do produto, adimensional; t: tempo de secagem
\end{tabular}

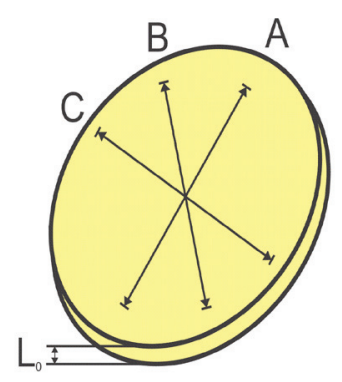

(a)

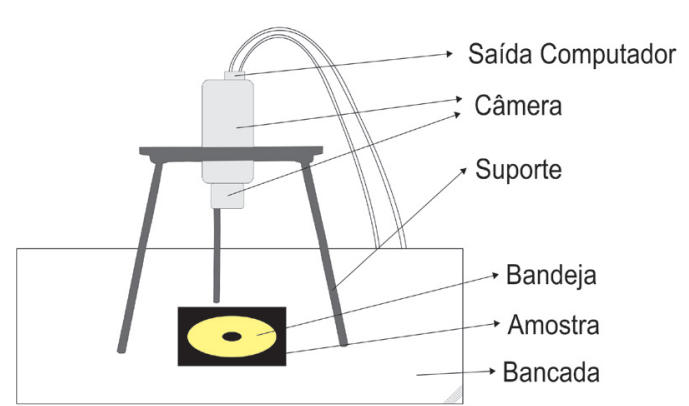

(b)

Figura 1. Coordenadas aferidas para a obtenção dos volumes das amostras (a), sendo L a espessura da fatia e A, B e C os diâmetros aferidos, e aparato experimental para captura das imagens (b). 
Tabela 2. Modelos matemáticos utilizados para estimar o encolhimento, durante a secagem.

\begin{tabular}{llll}
\hline & Designação do modelo & \multicolumn{1}{c}{ Modelo } & Referência \\
\hline \multirow{2}{*}{ Linear } & Lozano & $\mathrm{S}=\mathrm{b}_{1} \mathrm{U}^{*}+\mathrm{b}_{2}$ & Queiroz \& Nebra (2001) \\
& McMinn \& Magee & $\mathrm{S}=\left(\mathrm{b}_{1} \mathrm{~T}+\mathrm{b}_{2}\right)+\left(\mathrm{b}_{3} \mathrm{~T}+\mathrm{b}_{4}\right) \mathrm{U}^{*}$ & McMinn \& Magee (1997) \\
\multirow{2}{*}{ Não linear } & Rovedo et al. & $\mathrm{S}=\mathrm{b}_{1}+\mathrm{b}_{2} \exp \left(-\mathrm{b}_{3} \mathrm{t}\right)$ & Rovedo et al. (1997) \\
& Ratti & $\mathrm{S}=\mathrm{b}_{1}+\mathrm{b}_{2} \mathrm{U}^{*}+\mathrm{b}_{3}\left(\mathrm{U}^{*}\right)^{2}+\mathrm{b}_{4}\left(\mathrm{U}^{*}\right)^{3}$ & Ratti (1994) \\
\hline
\end{tabular}

$\mathrm{S}$ : encolhimento (adimensional); $\mathrm{U}^{*}$ : teor de água do produto, decimal (b.s.); $\mathrm{T}$ : temperatura $\left({ }^{\circ} \mathrm{C}\right)$; t: tempo $(\mathrm{s}) ; \mathrm{b}_{1}, \mathrm{~b}_{2}, \mathrm{~b}_{3}, \mathrm{~b}_{4}$ : constantes do modelo (adimensional).

$$
\begin{aligned}
& \mathrm{P}=\frac{100}{\mathrm{n}} \sum_{\mathrm{i}=1}^{\mathrm{n}} \frac{|\mathrm{Y}-\hat{\mathrm{Y}}|}{\mathrm{Y}} \\
& \mathrm{SE}=\sqrt{\frac{\sum_{\mathrm{i}=1}^{\mathrm{n}}(\mathrm{Y}-\hat{\mathrm{Y}})^{2}}{\mathrm{GLR}}}
\end{aligned}
$$

em que $\mathrm{Y}=$ valor observado experimentalmente; $\hat{\mathrm{Y}}=$ valor predito pelo modelo; $\mathrm{n}=$ número de observações experimentais; GLR = grau de liberdade do modelo (número de observações menos o número de parâmetros do modelo).

Os valores de difusividade efetiva média de umidade foram determinados por meio da solução analítica da Lei de Fick, para a difusão de água líquida em um sólido, levando-se em consideração as condições do material em análise, conforme a seguinte equação:

$$
\mathrm{RU}=\left(\frac{8}{\pi^{2}}\right) * \sum_{\mathrm{n}=0}^{\infty} \frac{1}{(2 \mathrm{n}+1)^{2}} \exp \left[-(2 \mathrm{n}+1)^{2} * \pi^{2} * \frac{\mathrm{D}_{\mathrm{EF}} * \mathrm{t}}{4 * \mathrm{~L}_{0}^{2}}\right]
$$

em que $\mathrm{L}_{0}=$ espessura da camada de produto; $\mathrm{n}=$ número de termos; $\mathrm{D}_{\text {ef }}=$ difusividade efetiva; $\mathrm{t}=$ tempo de secagem.

A difusividade efetiva, considerando-se o encolhimento, foi determinada substituindo-se a razão de umidade do produto pela concentração de umidade (Park 1987), como se segue:

$\frac{C-C_{\varepsilon}}{C_{0}-C_{\theta}}=\left(\frac{8}{\pi^{2}}\right) * \sum_{n=0}^{\infty} \frac{1}{(2 n+1)^{2}} \exp \left[-(2 n+1)^{2} * \pi^{2} * \frac{D_{E F} * t}{4 * L_{0}^{2}}\right]$

sendo $\mathrm{C}=$ concentração de umidade, $\mathrm{X} / \mathrm{V}\left(\mathrm{g} \mathrm{H}_{2} \mathrm{O} \mathrm{g}^{-1}\right.$ $\left.\mathrm{ms}^{-1} \mathrm{~m}^{-3}\right) ; \mathrm{C}_{\mathrm{e}}=$ concentração de umidade de equilíbrio, $\mathrm{X}_{\mathrm{e}} / \mathrm{V}_{\mathrm{e}}\left(\mathrm{g} \mathrm{H}_{2} \mathrm{O} \mathrm{g}^{-1} \mathrm{~ms}^{-1} \mathrm{~m}^{-3}\right) ; \mathrm{C}_{0}=$ concentração de umidade inicial, $\mathrm{X}_{0} / \mathrm{V}_{0}\left(\mathrm{~g} \mathrm{H}_{2} \mathrm{O} \mathrm{g}^{-1} \mathrm{~ms}^{-1} \mathrm{~m}^{-3}\right) ; \mathrm{n}=$ número de termos.

A energia de ativação $\left(E_{a}\right)$ foi obtida a partir da dependência da difusividade efetiva $\left(D_{\text {ef }}\right)$ com a temperatura, pela seguinte equação, do tipo Arrhenius:

$$
D_{\text {ef }}=D_{0} \cdot e^{-\frac{E_{a}}{R \cdot T}}
$$

em que $\mathrm{D}_{0}=$ constante, $\mathrm{m}^{2} \mathrm{~s}^{-1} ; \mathrm{E}_{\mathrm{a}}=$ energia de ativação, $\mathrm{J} \mathrm{mol}^{-1} ; \mathrm{R}=$ constante universal dos gases, $8,314 \mathrm{~J} \mathrm{~mol}^{-1} \mathrm{~K}^{-1} ; \mathrm{T}=$ temperatura absoluta, $\mathrm{K}$.

\section{RESULTADOS E DISCUSSÃO}

Apresentam-se, na Figura 2, as curvas de secagem para as fatias de banana variedade Terra, às temperaturas de $40{ }^{\circ} \mathrm{C}, 50^{\circ} \mathrm{C}$ e $60^{\circ} \mathrm{C}$.

Analisando-se as curvas de razão de umidade (RU), percebe-se que o aumento da temperatura favorece o processo de transferência de energia na forma de calor para as amostras, diminuindo, consequentemente, o tempo necessário para o sólido atingir o equilíbrio dinâmico. $\mathrm{O}$ mesmo comportamento foi observado por Kaleemullah \& Kailappan (2006), Doymaz (2007) e Wang et al. (2007), estudando, respectivamente, as cinéticas de secagem de pimenta vermelha, feijão verde e maçã.

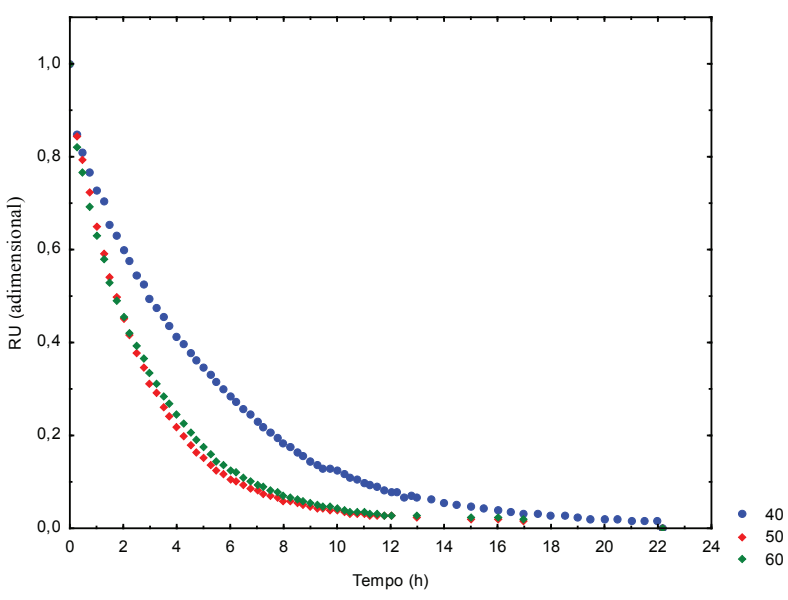

Figura 2. Curva de razão de umidade (RU), em função do tempo, para as secagens às temperaturas de $40{ }^{\circ} \mathrm{C}, 50^{\circ} \mathrm{C}$ e $60^{\circ} \mathrm{C}$ (Barra do Bugres, MT, 2011). 
Na Tabela 3, são apresentados os valores dos coeficientes de determinação $\left(\mathrm{R}^{2}, \%\right)$, erro médio estimado (SE, decimal) e erro médio relativo (P, \%), para os quatro modelos analisados para a secagen da matriz experimental.

Os modelos Midilli \& Kucuk e Thompson apresentaram ajustes com elevados coeficientes de determinação $\left(\mathrm{R}^{2}\right)$, superiores a $99 \%$, e baixo erro médio relativo $(\mathrm{P})$, ou seja, valores inferiores a $10 \%$, e baixo desvio padrão da estimativa (inferiores a 0,020) (Tabela 3). Segundo Goneli et al. (2009), tais resultados indicam uma representação satisfatória do processo de secagem.

Apesar de o modelo de Thompson ser adequado para a representação da cinética de secagem de fatias de banana variedade Terra, o modelo de Midilli \& Kucuk foi o escolhido, devido à facilidade de sua aplicação (Gurjão 2006). Resultado semelhante foi observado por Wang et al. (2007), Menges \& Ertekin (2006), Sacilik \& Konuralp (2006) e Gurjão (2006), os quais analisaram, respectivamente, a cinética de secagem de maçã, maçã variedade ouro, tomate orgânico e polpa de tamarindo.

Analisado-se os parâmetros estimados (Tabela 4), observa-se que a magnitude da constante de secagem (k) para o modelo de Midilli \& Kucuk, que representa o efeito das condições externas de secagem, aumenta com a elevação da temperatura de secagem.

$\mathrm{O}$ parâmetro $\mathrm{k}$ aumentou com a elevação da temperatura do ar de secagem, pois, quanto maior a temperatura de secagem, mais facilidade o alimento terá em perder água livre. O mesmo ocorreu com o parâmetro n. Já os parâmetros A e B são constantes do modelo e não possuem significado físico.

Resultado semelhante foi encontrado por Menges \& Ertekin (2006), Wang et al. (2007), Park et al. (2001) e Akpinar et al. (2003), ao estudarem, respectivamente, a secagem de maçã dourada, polpa de maçã, pera e pimentão. Segundo Babalis \& Belessiotis (2004), a constante de secagem (k) pode ser utilizada como aproximação para caracterizar o efeito da temperatura na difusividade efetiva de umidade, no processo de secagem.

Os valores dos coeficientes de determinação $\left(\mathrm{R}^{2}\right)$, desvio padrão da estimativa (SE) e erro médio relativo $(\mathrm{P})$ dos modelos matemáticos de encolhimento podem ser observados na Tabela 5 .

Pode-se verificar, na Tabela 4, que todos os modelos matemáticos para o encolhimento obtiveram adequado ajuste aos dados experimentais, pois apre-
Tabela 3. Valores do coeficiente de determinação $\left(\mathrm{R}^{2}\right)$, desvio padrão da estimativa (SE) e erro médio relativo $(\mathrm{P})$ calculados para a verificação de ajuste dos modelos matemáticos aos valores experimentais obtidos nas secagens a $40{ }^{\circ} \mathrm{C}, 50{ }^{\circ} \mathrm{C}$ e $60{ }^{\circ} \mathrm{C}$ (Barra do Bugres, MT, 2011).

\begin{tabular}{cccrrr}
\hline & & $\begin{array}{c}\text { Aproximação } \\
\text { da difusão }\end{array}$ & $\begin{array}{c}\text { Dois } \\
\text { termos }\end{array}$ & $\begin{array}{c}\text { Midilli \& } \\
\text { Kucuk }\end{array}$ & Thompson \\
\hline \multirow{2}{*}{$\mathrm{R}^{2}$} & $40^{\circ} \mathrm{C}$ & 99,520 & 99,980 & 99,990 & 99,860 \\
$(\%)$ & $50^{\circ} \mathrm{C}$ & 99,650 & 99,470 & 99,990 & 99,980 \\
& $60^{\circ} \mathrm{C}$ & 99,810 & 99,790 & 99,900 & 99,870 \\
\hline \multirow{2}{*}{$\mathrm{P}$} & $40^{\circ} \mathrm{C}$ & 4,036 & 0,701 & 0,839 & 3,285 \\
$(\%)$ & $50^{\circ} \mathrm{C}$ & 12,563 & 20,316 & 2,969 & 3,247 \\
& $60{ }^{\circ} \mathrm{C}$ & 6,954 & 11,714 & 8,713 & 8,992 \\
\hline \multirow{3}{*}{$\mathrm{SE}$} & $40^{\circ} \mathrm{C}$ & 0,021 & 0,004 & 0,003 & 0,011 \\
& $50^{\circ} \mathrm{C}$ & 0,020 & 0,024 & 0,003 & 0,004 \\
& $60^{\circ} \mathrm{C}$ & 0,015 & 0,016 & 0,011 & 0,012 \\
\hline
\end{tabular}

Tabela 4. Parâmetros obtidos do modelo de Midilli \& Kucuk, ajustados aos dados de secagem de banana variedade Terra, para as temperaturas de $40{ }^{\circ} \mathrm{C}, 50{ }^{\circ} \mathrm{C}$ e $60{ }^{\circ} \mathrm{C}$ (Barra do Bugres, MT, 2011).

\begin{tabular}{ccccc}
\hline Temperatura & $\mathrm{k}$ & $\mathrm{A}$ & $\mathrm{n}$ & $\mathrm{B}$ \\
\hline $40^{\circ} \mathrm{C}$ & 0,322352 & 0,996660 & 0,625734 & $-0,007516$ \\
$50{ }^{\circ} \mathrm{C}$ & 0,542943 & 0,992946 & 0,767042 & $-0,000562$ \\
$60^{\circ} \mathrm{C}$ & 0,586712 & 0,977306 & 0,869598 & $-0,000676$ \\
\hline
\end{tabular}

$\mathrm{k}$ : coeficiente de secagem, $\mathrm{h}^{-1} ; \mathrm{A}, \mathrm{B}, \mathrm{n}$ : constantes dos modelos.

Tabela 5. Valores dos coeficientes de determinação $\left(\mathrm{R}^{2}\right)$, desvio padrão da estimativa (SE) e erros médios relativos (P) calculados para a verificação do ajuste dos modelos matemáticos de encolhimento (Barra do Bugres, MT, 2011).

\begin{tabular}{cccccc}
\hline & & \multirow{2}{*}{ Lozano } & $\begin{array}{c}\text { McMinn \& } \\
\text { Magee }\end{array}$ & $\begin{array}{c}\text { Rovedo, } \\
\text { Suàrez e Viollaz }\end{array}$ & \multirow{2}{*}{ Ratti } \\
\hline \multirow{2}{*}{$\mathrm{R}^{2}$} & $40{ }^{\circ} \mathrm{C}$ & 99,170 & 99,170 & 99,130 & 99,220 \\
$(\%)$ & $50{ }^{\circ} \mathrm{C}$ & 98,170 & 98,110 & 97,110 & 98,780 \\
& $60{ }^{\circ} \mathrm{C}$ & 98,070 & 98,070 & 97,580 & 99,090 \\
\hline \multirow{2}{*}{$\mathrm{P}$} & $40{ }^{\circ} \mathrm{C}$ & 0,713 & 0,713 & 0,678 & 0,676 \\
$(\%)$ & $50{ }^{\circ} \mathrm{C}$ & 1,062 & 1,062 & 1,236 & 0,847 \\
& $60{ }^{\circ} \mathrm{C}$ & 0,903 & 0,903 & 0,898 & 0,627 \\
\hline \multirow{3}{*}{$\mathrm{SE}$} & $40^{\circ} \mathrm{C}$ & 0,008 & 0,008 & 0,008 & 0,008 \\
& $50^{\circ} \mathrm{C}$ & 0,010 & 0,010 & 0,012 & 0,008 \\
& $60^{\circ} \mathrm{C}$ & 0,009 & 0,009 & 0,010 & 0,006 \\
\hline
\end{tabular}

sentaram elevados coeficientes de determinação $\left(\mathrm{R}^{2}\right)$, baixo erro médio relativo $(\mathrm{P})$ e baixo desvio padrão da estimativa (SE). Porém, o modelo de Ratti, por ser uma equação não linear e com maior número de parâmetros, apresenta ajuste com maior $\mathrm{R}^{2}$, menor $\mathrm{P}$ 
e menor SE, em relação aos demais modelos, estando de acordo com os resultados encontrados por Goneli et al. (2009). Segundo os autores, tais resultados indicam uma representação satisfatória para o processo de encolhimento.

Diante dos parâmetros do modelo de Ratti (Tabela 6), pode-se verificar a dependência do encolhimento ao teor de umidade, sendo ela um polinômio de terceira ordem, ou seja, na primeira etapa de secagem, o encolhimento tem uma dependência linear com a remoção de água. Posteriormente, a quantidade de água vai diminuindo e a temperatura de transição vítrea (Tg) aumenta e, com isso, o material passa de um estado elástico (etapa linear) para um estado inteiramente rígido e não flexível, diminuindo, assim, a razão de encolhimento (dependência polinomial), até atingir a umidade de equilíbrio (Lopes 2013). Ochoa et al. (2007), ao analisarem o fenômeno do encolhimento de frutos de cereja doce inteiros, observaram o mesmo comportamento.

Tabela 6. Parâmetros obtidos para o modelo de Ratti, ajustados aos dados experimentais obtidos nas secagens a $40{ }^{\circ} \mathrm{C}$, $50{ }^{\circ} \mathrm{C}$ e $60^{\circ} \mathrm{C}$ (Barra do Bugres, MT, 2011).

\begin{tabular}{ccccrr}
\hline Modelo & $\mathrm{T}\left({ }^{\circ} \mathrm{C}\right)$ & $\mathrm{b}_{1}$ & $\mathrm{~b}_{2}$ & \multicolumn{1}{c}{$\mathrm{b}_{3}$} & \multicolumn{1}{c}{$\mathrm{b}_{4}$} \\
\hline \multirow{3}{*}{ Ratti } & 40 & 0,682 & 0,180 & 0,354 & $-0,212$ \\
& 50 & 0,745 & 0,436 & $-0,561$ & 0,388 \\
& 60 & 0,773 & 0,374 & $-0,533$ & 0,393 \\
\hline
\end{tabular}

$\mathrm{T}$ : temperatura; $\mathrm{b}_{1}, \mathrm{~b}_{2}, \mathrm{~b}_{3}, \mathrm{~b}_{4}$ : constantes do modelo (adimensional).
Isso pode ser verificado na Figura 3, onde são apresentados os valores observados para encolhimento $(\mathrm{S})$ da massa, em função da razão de umidade adimensional, para o modelo de Ratti.

Além da redução da área, a secagem conferiu coloração característica às amostras, possivelmente devido à concentração de solutos e à reação de Maillard (Celestino 2010).

Com os valores médios dos volumes iniciais $\left(\mathrm{V}_{0}\right)$ e dos volumes de equilíbrio $\left(\mathrm{V}_{\mathrm{e}}\right)$, para as amostras de fatias de banana variedade Terra, foi possível calcular a redução do volume (RV), sendo este expresso em percentual, bem como os valores obtidos por meio do software (Tabela 7).

Pode-se verificar, na Tabela 7, que a redução do volume (RV) apresentou comportamento inversamente proporcional, em relação à temperatura. Segundo Park et al. (2001), tal fenômeno ocorre devido à redução rápida do teor de umidade sob altas temperaturas, o que ocasiona rápida transição do estado elástico para o estado vítreo, acarretando redução na taxa de encolhimento.

A diferença entre a variação das $\mathrm{D}_{\text {ef }}$ encontradas durante o processo de secagem da banana pode ter ocorrido pelo fato de que a captura dos dados pela imagem leva em consideração toda a área da fatia (Tabela 8). Já quando se aferem os dados com paquímetro, em três diferentes posições, pode ocorrer erro, pois o encolhimento da fatia não é uniforme.

A influência do encolhimento nos valores de difusividade mássica pode ser verificada devido à

Tabela 7. Valores dos volumes iniciais, de equilíbrio e redução do volume (RV), com dados aferidos por paquímetro(1) e captados por imagens $^{(2)}$, para as temperaturas estudadas (Barra do Bugres, MT, 2011).

\begin{tabular}{ccccccc}
\hline $\mathrm{T}\left({ }^{\circ} \mathrm{C}\right)$ & $\mathrm{V}_{0}^{(1)}\left(\mathrm{m}^{3}\right)$ & $\mathrm{V}_{\mathrm{e}}^{(1)}\left(\mathrm{m}^{3}\right)$ & $\mathrm{RV}^{(1)}(\%)$ & $\mathrm{V}_{0}^{(2)}\left(\mathrm{m}^{3}\right)$ & $\mathrm{V}_{\mathrm{e}}^{(2)}\left(\mathrm{m}^{3}\right)$ & $\mathrm{RV}^{(2)}(\%)$ \\
\hline 40 & $4,31 \times 10^{-6}$ & $3,38 \times 10^{-6}$ & 21,65 & $4,91 \times 10^{-6}$ & $3,54 \times 10^{-6}$ & 27,74 \\
50 & $3,63 \times 10^{-6}$ & $2,92 \times 10^{-6}$ & 19,46 & $4,71 \times 10^{-6}$ & $3,58 \times 10^{-6}$ & 24,08 \\
60 & $3,88 \times 10^{-6}$ & $3,19 \times 10^{-6}$ & 17,74 & $5,66 \times 10^{-6}$ & $4,39 \times 10^{-6}$ & 22,50 \\
\hline
\end{tabular}

$\mathrm{V}_{\mathrm{e}}$ : volume de equilíbrio $\left(\mathrm{m}^{3}\right) ; \mathrm{T}$ : temperatura.

Tabela 8. Valores de difusividade mássica estimados pelo modelo difusional com e sem a consideração do encolhimento (Barra do Bugres, MT, 2011)

\begin{tabular}{cccccc}
\hline $\mathrm{T}\left({ }^{\mathrm{o}} \mathrm{C}\right)$ & $\mathrm{D}_{\mathrm{ef}}\left(\mathrm{m}^{2} \mathrm{~s}^{-1}\right)^{(1)}$ & $\mathrm{D}_{\mathrm{ef}}\left(\mathrm{m}^{2} \mathrm{~s}^{-1}\right)^{(2)}$ & $\mathrm{D}_{\mathrm{ef}}\left(\mathrm{m}^{2} \mathrm{~s}^{-1}\right)^{(3)}$ & $\mathrm{RD}^{(1)}\left(\mathrm{D}_{\text {ef }}, \%\right)$ & $\mathrm{RD}^{(2)}\left(\mathrm{D}_{\mathrm{ef}}, \%\right)$ \\
\hline 40 & $5,64 \times 10^{-10}$ & $4,34 \times 10^{-10}$ & $4,29 \times 10^{-10}$ & 23,04 & 23,94 \\
50 & $8,79 \times 10^{-10}$ & $7,59 \times 10^{-10}$ & $7,06 \times 10^{-10}$ & 13,65 & 19,68 \\
60 & $9,86 \times 10^{-10}$ & $8,85 \times 10^{-10}$ & $8,63 \times 10^{-10}$ & 10,24 & 12,47 \\
\hline
\end{tabular}

Redução da difusividade (\%): (1) sem considerar o encolhimento; ${ }^{(2)}$ considerando-se o encolhimento com resultados obtidos com os dados do paquímetro; ${ }^{(3)}$ considerandose o encolhimento com resultados obtidos com os dados das imagens. 


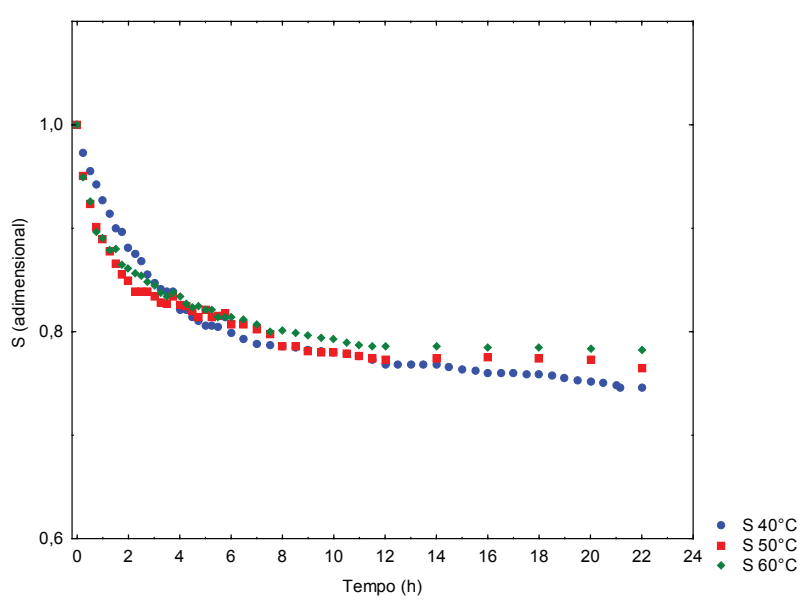

Figura 3. Valores observados para o encolhimento de fatias de banana variedade Terra submetidas a secagem às temperaturas de $40{ }^{\circ} \mathrm{C}, 50{ }^{\circ} \mathrm{C}$ e $60{ }^{\circ} \mathrm{C}$, para o modelo de Ratti (Barra do Bugres, MT, 2011).

variação da redução de difusividade (RD), que foi de 10,04-23,04\% (paquímetro) e 12,47-23,94\% (imagem).

Os valores encontrados para a energia de ativação $\left(\mathrm{E}_{\mathrm{a}}\right)$ foram de $24,07 \mathrm{~kJ} \mathrm{~mol}^{-1}$, sem considerar o encolhimento, e de $30,71 \mathrm{~kJ} \mathrm{~mol}^{-1}$ e $30,17 \mathrm{~kJ} \mathrm{~mol}^{-1}$, utilizando-se os valores de difusividade com o fenômeno do encolhimento com resultados dos dados do paquímetro e imagem, respectivamente. Ou seja, considerando-se o encolhimento, a energia de ativação sofreu aumento de, aproximadamente, $20 \%$. Tais valores encontram-se dentro da faixa apresentada como aceitável por Zogzas et al. (1996), para produtos agrícolas, que varia de $12,7 \mathrm{~kJ} \mathrm{~mol}^{-1}$ a $110 \mathrm{~kJ} \mathrm{~mol}^{-1}$.

\section{CONCLUSÕES}

1. A temperatura influencia no processo de secagem, pois, quanto maior a temperatura de secagem, mais rápido a banana atinge seu equilíbrio termodinâmico.

2. A redução de volume apresenta comportamento inversamente proporcional, em relação às temperaturas estudadas.

\section{REFERÊNCIAS}

AFONSO JÚNIOR, P. C.; CORRÊA, P. C. Comparação de modelos matemáticos para descrição da cinética de secagem em camada fina de sementes de feijão. Revista
Brasileira de Engenharia Agrícola e Ambiental, Campina Grande, v. 3, n. 3, p. 349-353, 1999.

AKPINAR, E. K.; BICER, Y.; YILDIZ, C. Thin layer drying of red pepper. Journal of Food Engineering, Oxford, v. 59, n. 1, p. 99-104, 2003.

BABALIS, S. J.; BELESSIOTIS, V. G. Influence of the drying conditions on the drying constants and moisture diffusivity during the thin-layer drying of figs. Journal of Food Engineering, Oxford, v. 65, n. 3, p. 449-58, 2004.

BATISTA, L. M. Secagem de quitosana obtida a partir de resíduos de camarão: análise da cinética de secagem considerando encolhimento. 2004. 121 f. Dissertação (Mestrado em Engenharia e Ciência de Alimentos) Fundação Universidade do Rio Grande, Rio Grande, 2004.

BORGES, S. V. et al. Secagem de fatias de abóboras (Curcubit moschata L.) por convecção natural e forçada. Ciência e Tecnologia de Alimentos, Campinas, v. 28, n. 2, p. 245-251, 2008.

BRUNINI, M. A.; DURIGAN, J. F.; OLIVEIRA, A. L. Avaliação das alterações em polpa de manga "Tommy Atkins" congelada. Revista Brasileira de Fruticultura, Jaboticabal, v. 24, n. 3, p. 651-653, 2002.

CElestino, S. M. C. Principios de secagem de alimentos. Planaltina, DF: Embrapa Cerrados, 2010.

CHITARRA, M. I. F.; CHITARRA, A. B. Pós-colheita de frutos e hortaliças: fisiologia e manuseio. Lavras: FaepeESAL, 1990.

DANDAMRONGRAK, R.; YOUNG, G.; MASON, R. Evaluation of various pre-treatmentes for the dehydration of banana and selection of suitable drying models. Journal of Food Engineering, Oxford, v. 55, n. 2, p. 139-146, 2002.

DANTAS, T. N. P. et al. Estudo da transferência de calor e massa e da cinética de secagem em placas planas de batata inglesa (Solanum Tuberosum L.). In: CONGRESSO BRASILEIRO DE ENGENHARIA QUÍMICA EM INICIAÇÃO CIENTÍFICA, 8., 2009, Uberlândia. Disponível em: <http://www.cobeqic2009.feq.ufu.br/ uploads/media/105621281.pdf>. Acesso em: 09 jan. 2015.

DOYMAZ, I. Air-drying characteristics of tomatoes. Journal of Food Engineering, Oxford, v. 78, n. 4, p. 12911297, 2007.

DROUZAS, A. E.; SCHUBERT, H. Microwave application in vacuum drying of fruit. Journal of Food Engineering, Oxford, v. 28, n. 2, p. 203-209, 1996.

ERTEKIN, C.; YALDIZ, O. Drying of eggplant and selection of a suitable thin layer drying model. Journal of Food Engineering, Oxford, v. 63, n. 3, p. 349-359, 2004.

GONELI, A. L. D. et al. Cinética de secagem dos grãos de café descascados em camada delgada. Revista Brasileira de Armazenamento, Viçosa, n. 11, p. 74-80, 2009. 
GURJÃO, K. C. O. Desenvolvimento, armazenamento e secagem de tamarindo (Tamarindus indica L.). 2006. $165 \mathrm{f}$. Tese (Doutorado em Agronomia) - Universidade Federal da Paraíba, Areia, 2006.

JESUS, C. de et al. Caracterização física e química de frutos de diferentes genótipos de bananeira. Bragantia, Campinas, v. 63, n. 3, p. 315-323, 2004.

KALEEMULLAH, S.; KAILAPPAN, R. Modelling of thin-layer drying kinetics of red chillies. Journal of Food Engineering, Oxford, v. 76, n. 4, p. 531-537, 2006.

KATEKAWA, M. E. Análise teórico-experimental do encolhimento: estudo da secagem de banana. 2006. $151 \mathrm{f}$. Tese (Doutorado em Engenharia Química) - Universidade Estadual de Campinas, Campinas, 2006.

LAHSASNI, S. et al. Drying kinetcs of prickly pear fruit (Opuntia ficus indica). Journal of Food Engineering, Oxford, v. 61, n. 2, p. 173-179, 2004.

LOPES, F. J. Estudo do fenômeno de encolhimento na secagem convectiva de abacaxi com aplicação de microondas. 2013. 143 f. Dissertação (Mestrado em Produção Vegetal) - Universidade Estadual do Norte Fluminense Darcy Ribeiro, Campos dos Goytacazes, 2013.

MCMINN, W. A. M.; MAGEE, T. R. A. Physical characteristics of dehydrated potatoes: part I. Journal of Food Engineering, Oxford, v. 33, n. 1-2, p. 37-48, 1997.

MENGES, H. O.; ERTEKIN, C. Mathematical modeling of thin layer drying of Golden apples. Journal of Food Engineering, Oxford, v. 77, n. 1, p. 119-125, 2006.

MIDILLI, A.; KUCUK, H.; YAPAR, Z. A. New model for single-layer drying. Drying Technology, Nigde, v. 20, n. 7, p. 1503-1513, 2002.

OCHOA, M. R. et. al. Analysis of shrinkage phenomenon of whole sweet cherry fruits (Prunus avium) during convective dehydration with very simple models. Journal of Food Engineering, Oxford, v. 79, n. 2, p. 657-661, 2007.

PARANÁ. Secretaria de Estado da Agricultura e do Abastecimento (SEAB). Fruticultura: análise da conjuntura agropecuária. 2012. Disponível em: $<$ http://www.agricultura.pr.gov.br/arquivos/File/deral/ Prognosticos/fruticultura_2012_13.pdf $>$. Acesso em: 09 jan. 2015.

PARK, K. J. Estudo comparativo do coeficiente de difusão sem e com encolhimento durante a secagem. 1987. $54 \mathrm{f}$. Tese (Livre Docência em Fenômenos de Transporte) Faculdade de Engenharia de Alimentos, Universidade Estadual de Campinas, Campinas, 1987.

PARK, K. J.; YADO, M. K. M.; BROD, F. P. R. Estudo de secagem de pera Bartlett (Pyrus sp.) em fatias. Ciência e Tecnologia de Alimentos, Campinas, v. 21, n. 3, p. 288292, 2001.

PONTES, S. F. O. et al. Secagem e avaliação sensorial de banana da terra. Revista Brasileira de Produtos Agroindustriais, Campina Grande, v. 9, n. 2, p. 143-148, 2007.

RATTI, C. Shrinkage during drying of foodstuffs. Journal of Food Engineering, Oxford, v. 23, n. 1, p. 91-105, 1994.

REIS, R. C. et al. Modelagem matemática da secagem de pimenta Cumari do Pará. Revista Brasileira de Engenharia Agrícola e Ambiental, Campina Grande, v. 15, n. 4, p. $347-$ 353, 2011.

ROCA, E. et al. Effective moisture diffusivity modelling versus food structure and hygroscopicity. Food Chemistry, Montpellier, v. 106, n. 4, p. 1428-1437, 2008.

ROVEDO, C. O.; SUAREZ, C.; VIOLLAZ, P. E. Kinetics of forced convective air drying of potato and squash slabs. Food Science and Technology International, Buenos Aires, v. 3, n. 4, p. 251-261, 1997.

QUEIROZ, M. R; NEBRA, S. A. Theoretical and experimental analysis of the drying kinetics of bananas. Journal of Food Engineering, Oxford, v. 4, n. 2, p. $127-$ 132, 2001.

SACILIK, K.; KONURALP, A. E. The thin layer drying characteristics of organic apple slices. Journal of Food Engineering, Oxford, v. 73, n. 3, p. 281-289, 2006.

SHARMA, G. P; PRASAD, S. Effective moisture diffusivity of garlic cloves undergoing microwaveconvective drying. Journal of Food Engineering, Oxford, v. 65 , n. 4, p. 609-617, 2004.

SILVA, A. S. et al. Cinética de secagem em camada fina da banana maçã em secador de leito fixo. Revista Brasileira de Produtos Agroindustriais, Campina Grande, v. 11, n. 2, p. 129-136, 2009.

SOUSA, P. H. M. Desidratação osmótica de banana com e sem vácuo com complemento de secagem em estufa de circulação de ar. 2002. 82 f. Dissertação (Mestrado em Tecnologia de Alimentos) - Universidade Federal do Ceará, Fortaleza, 2002.

WANG, Z. et al. Mathematical modeling on hot air drying of thin layer apple pomace. Received Food Research International, Beijing, v. 40, n. 1, p. 39-46, 2007.

ZOGZAS, N. P.; MAUROULIS, Z. B.; MARINOSKOURIS, D. Moisture diffusivity data compilation in foodstuffs. Drying Technology, Athens, v. 14, n. 10, p. 2225-2253, 1996. 